\title{
The Simulation about Electric-field Distribution and Gain Characteristic of GEM Detector by Maxwell and Garfield
}

\author{
Ai-xing Wang ${ }^{1,2, a}$, \\ ${ }^{2}$ College of Science, \\ East China Institute of Technology, \\ Nanchang 330013, China \\ axingxing_fz@sina.com
}

\author{
Qianglin Wei ${ }^{1, b}$, Yibao Liu*1,c \\ ${ }^{1}$ Engineering Research Center of Nuclear Technology \\ Application (East China Institute of Technology), \\ Ministry of Education, \\ Nanchang, 330013, China \\ bqlwei11@ecit.cn, ${ }^{c}$ liuyb01@mails.tsinghua.edu.cn
}

\begin{abstract}
The minimum unit geometric model of signal GEM(Gas Electron Multiplier) detector has been build, and the Maxwell v11 program is used to calculate the electric potential, electric field, potential value of triangular meshes of detector, and the hemitropic faces and electric field intensity vector graphs can be drawn at the same time; Then the calculated result is imported into Garfield program, and simulate the avalanche drift process of single electron and multiple electrons under the role of electric field by Garfield program. In addition, the gas effective gain that varies with the change of the proportion of mixed gas, collecting field, drift field, GEM membrane electrode voltages are also be simulated and discussed.
\end{abstract}

Keywords-GEM detector; electric-field distribution; ain characteristic; maxwell and Garfield.

\section{INTRODUCTION}

It is acknowledged that detectors are widely used in many facet of nuclear energy, such as detecting different kinds of tiny particles, identifying the radioactive fission products, recording information such as particle track, time flight and particle physical properties .As people learn more about our material word, the study of detectors goes deeper $^{[1]}$. Gas electron multiplier detector is first invented by CERN in recent decade ${ }^{[2]}$, and now it is widely regarded as a new excellent gas detector. What appeals to most people is that GEM has a striking characteristic--- It can get a relatively large signs by changing the voltage of the porous copper plating on conductive membrane.

The Performance of GEM detector is generally accepted very excellent, but the process requirement of GEM membrane's production is very strict, and only few European laboratory can produce it up to now. This delicate detector have many attractive advantages, which can be described as follows: High price, easy be damaged by firelighter, and the largest size is $30 \mathrm{~cm} * 30 \mathrm{~cm}$. what's more, the secondary and triple GEM detector have also been widely used in all kinds of particle beam test(such as particle track measurement $t^{[3]}$, TCP read out ${ }^{[4,5]}$, fast trigger $^{[6]}$, neutron imaging ${ }^{[7,8]}$, photon detection ${ }^{[9]}$ etc) especially high-energy physics and particle physics and some other relevant aspects.

\section{INTRODUCTION OF TWO SIMULATION SOFTWARE: MAXWELL V11 AND GARFIELD}

Maxwell is a sort of finite element simulation software, which has wide application in engineering electromagnetic field. This kind of software can subtly convert the complex electromagnetic calculation to a gigantic matrix in $\mathrm{FE}$ discrete forms on the basis of the Maxwell differential equations ${ }^{[10]}$. Fig. 1 shows to us some brief operation steps of Maxwell simulation ${ }^{[11]}$. Garfield program, on the contrary, are mainly used to simulate the interaction between ions or free electrons and gas molecules in some traditional detectors $^{[12]}$ (Eg.ionization chamber, multi-wire proportional chamber). Due to this program itself without the function of calculating the electric field intensity, It must utilize the Ansoft Maxwell software ${ }^{[13]}$ to reckon the electric potential, electric field, and potential value. Meanwhile, the calculated results will be imported to the Garfield via interface program.

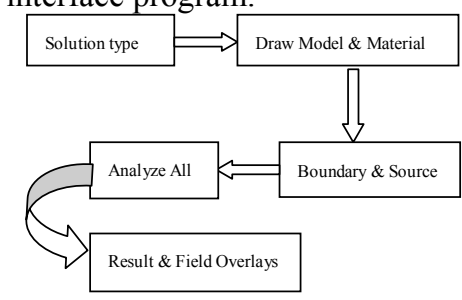

Fig.1. Maxwell simulation procedures

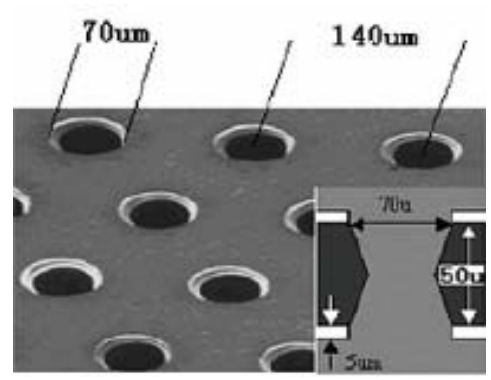

Fig.2. Maxwell simulation procedures 


\section{THE SIMULATION AND ANALYSIS OF GEM DETECTORS}

\section{A. Modeling a geometric unit of GEM membrane}

Usually speaking, the Copper clad of a standard GEM membrane materials is $5 \mu \mathrm{m}$, the thickness of the Kapton film is $50 \mu \mathrm{m}$, the hole spacing is $140 \mu \mathrm{m}$, and the aperture is $70 \mu \mathrm{m}$.

GEM membrane plays the most important role in GEM detector. What deserves to be mentioned is that the shape of micro-porous GEM membrane can determine the electric field distribution in tiny pores, which can exert a further significant effect on gas gain. In addition, considering GEM membrane's structure is a cyclical repeat unit, So during the process of simulation, we must take into account the symmetry of holes, the computational efficiency and the influences brought by electrostatic shielding on copper film. A minimal geometrical unit was sketched in Fig. 3.

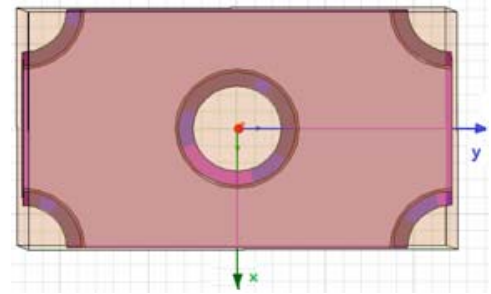

Fig.3. GEM membrane smallest geometric unit membrane

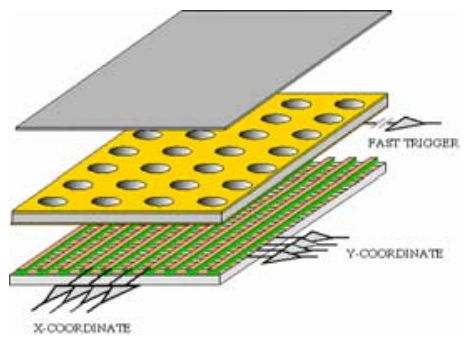

Fig.4. The spatial structure diagram of the single GEM detector

In order to build a minimum unit of GEM membrane, we should choose a biconical micro-porous structure with a high gain. Four parts are needed to build in this process: Two cones and two cylinders. And the other four $1 / 4$ holes can also be built by the same method. Just as Fig.3 shown; we can finally set up a smallest unit of GEM membrane model with Maxwell software (see Fig. 4).

\section{B. Modeling a geometric unit of GEM detector}

As is vividly sketched, a spatial structure of the single GEM detector was presented in Fig.4. And on the basis of smallest selected unit module, we can easily build a minimum unite model. After that, for the purpose of modeling a spatial structure(see Fig.5),then we need to establish a rectangle box with two thin parallel plates up and down and remove one minimum geometric unit of the GEM membrane inside of the box. By using Maxwell software, we can get the geometric potential and $3 \mathrm{~d}$ grid data directly. And the $3 \mathrm{~d}$ geometric potential diagrams and field vector diagrams can also been graphed in the post-processing module, as sketched in Fig. 6.

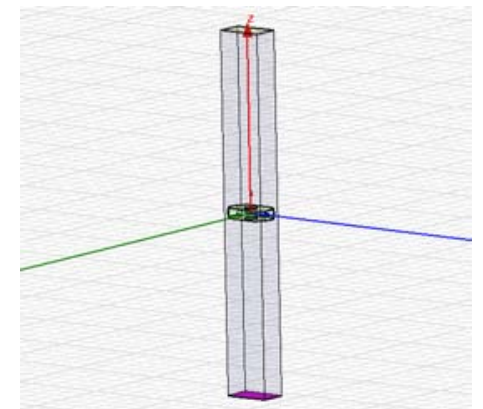

Fig.5. The smallest geometry model of single GEM detector signal

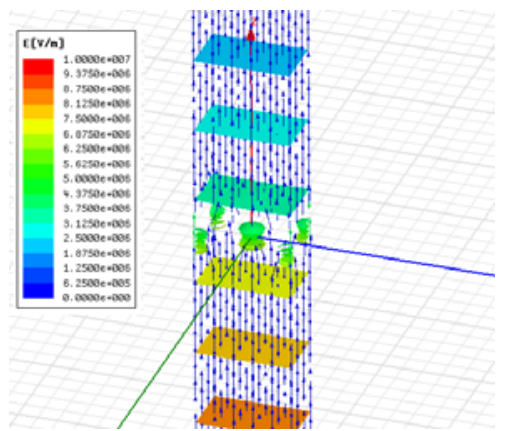

Fig.6. Electric field intensity vector of GEM geometry model

\section{The performance simulation of GEM detector}

1) The simulation of electron avalanche

When two $\mathrm{Cu}$ electrodes are placed in different potentials, a strong electric field will be formed inside of the holes. Just as the calculated electric field distribution(see Fig.7) demonstrated, free electrons produced on the surface of attachment(the drift region) will float into the holes under the control of electric field , and then they will undergo a gas avalanche amplification in drift field. In this process, a large quantities of electrons will released, and ultimately gathered below the GEM membrane, sketched in Fig.7.

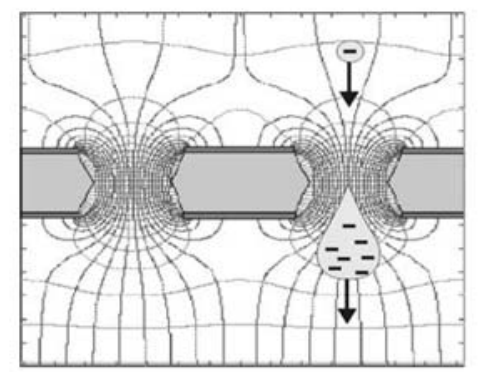

Fig.7. The electric field lines on the GEM section

The electron avalanche simulation is a complex process, Firstly; we should ascertain the specified boundary and conditions of materials. Take Kapton for example, its vacuum permittivity is about 2.89 and electrical conductivity is about $6.67 * 10^{-16} \mathrm{~s} / \mathrm{m}$. Using mixed $\operatorname{gas}\left(80 \% \mathrm{Ar}+20 \% \mathrm{CO}_{2}\right)$ as its working medium(the composition and proportion can be combined according to 
the experiment ), room temperature as its working environment, and 1 standard atmospheric pressure as its working pressure, a approximate vacuum environment in the analysis of electrostatic field will be created. For example, if we set the voltage of drift field to $1500 / \mathrm{cm}$, transmission field to $3600 / \mathrm{cm}$, entrance window to $-910 \mathrm{~V}$, and the upper copper GEM membrane $-760 \mathrm{~V}$, the lower copper membrane $-360 \mathrm{~V}$, the collecting plate potential $0 \mathrm{~V}$, then we can consequently reckon the corresponding

data: $\Delta U_{G}=400 \mathrm{~V}, \Delta U_{d}=150 \mathrm{~V}, \Delta U_{i}=360 \mathrm{~V}$.

Under the control of filed intensity, electrons are randomly moved. When they are accelerated to a certain speed, they will cause lots of ionization in the gas, which can described as "electrons avalanche" (see Fig.8). There are four kinds of probable circumstances in this situation:(a)If we press a certain difference between the top and the bottom of GEM membrane, electric field will increase sufficiently, and even break the limits of threshold(general is about $11 \mathrm{~K} / \mathrm{cm}$ ) that electrons avalanche can happen. However, if the voltage is lower than the threshold, even if the electrons migrate though the GEM membrane and be collected by plates, signals cannot be amplified (see Fig.8a).(b) some electrons are absorbed by the GEM membrane or gas molecules before the intensity entering the GEM film hole, which resulting the avalanche unable to amplified (see Fig.8b);(c) avalanches can be triggered due to strong field intensity, the (Fig.8c);(d) A multiple avalanches occurred with a host of different field intensity(Fig.8d).

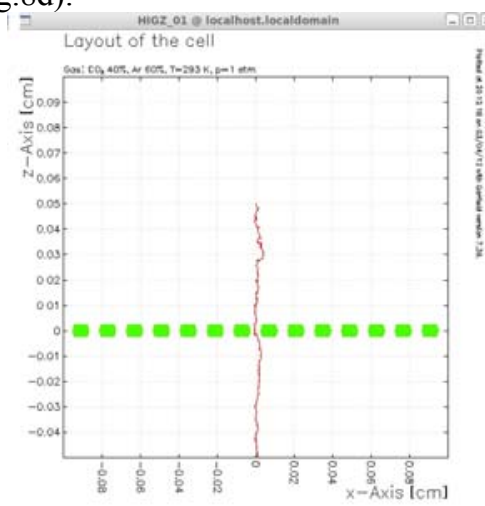

(a)

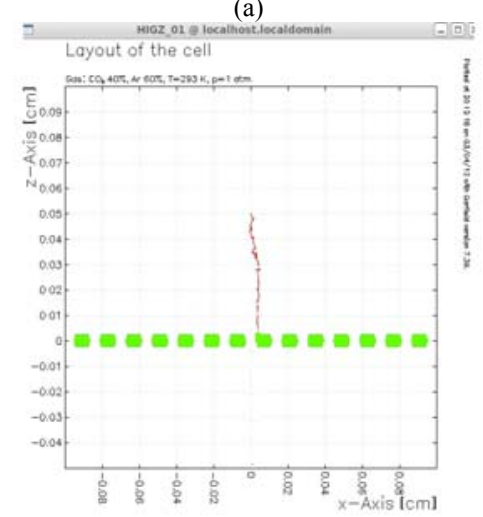

(b)

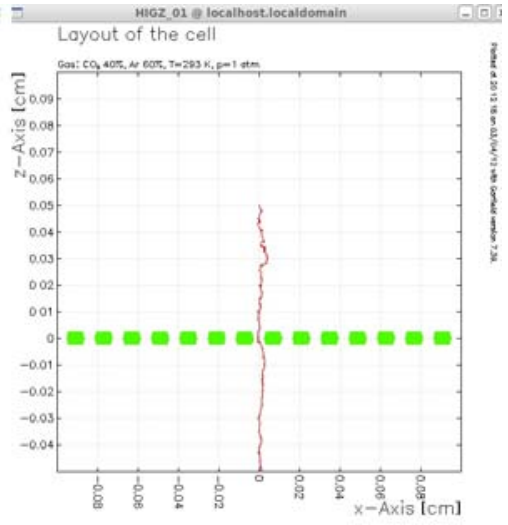

(c)

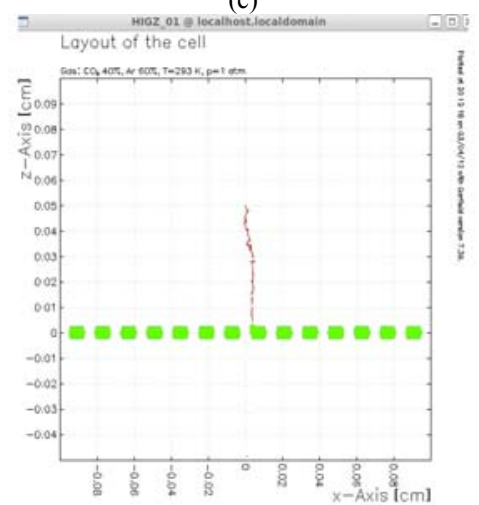

(d)

Fig.8. Electron avalanche process under the action of electric field

\section{2) The simulation of effective gain}

As $\mathrm{CO}_{2}$ concentration increases, the effective gain decreases approximate linearly (see Fig.9). Actually, the increase of $\mathrm{CO}_{2}$ concentration can exert some inhibitory influences on "avalanche effect", and as the electronic pair constantly decreasing, the number of collected electrons is corresponding declined, which, on the contrary, will eventually cause detector deterioration. In addition, $\mathrm{CO}_{2}$ is a typical quenching gas, and it has capable of preventing gas discharges. Meanwhile, the constant increase of $\mathrm{CO}_{2}$ in gas can also bring advantages to detector resolution. If the $\mathrm{CO} 2$ concentration is low, weak quenching and multiple scattering will be led.

Besides, the increasing $\mathrm{CO}_{2}$ concentration can enhance a significant quenching effect. Therefore, in order to meet a higher counting rate and a more excellent energy resolution, for gas mixture $\left(\mathrm{Ar}+\mathrm{CO}_{2}\right)$, the $\mathrm{CO}_{2}$ concentration is best to set at $15 \% \sim 25 \%$.

When electrons are allowed to enter GEM microspores, it will trigger a gigantic avalanche due to a strong electric field.As a result, it may bring about an amplification effect. If we respectively set the electric field of drift and collecting area at $1.0 \mathrm{KV} / \mathrm{cm}$ and $3.6 \mathrm{KV} / \mathrm{cm}$, and the ratio for $\mathrm{Ar} / \mathrm{CO}_{2}$ up to $70 / 30$, we can observe that with the increase of $\Delta_{\mathrm{VGEM}}$, the electric field in GEM membrane will corresponding increase. This phenomenon can be explained as follows: as the electric field in the primary ionization is 
raised higher, more and more electric ion pairs will be produced in the process of colliding with Ar atom. Accounting that avalanche is a process that primary electrons can continue to cause further ionization in the gas, we can concluded that the increases of electric field may lead to a significant electron avalanche and a remarkable GEM detector effective gain (up to $10^{3}$ ). Fig. 10 shows to us the tendency of electrons under the function of electric field.

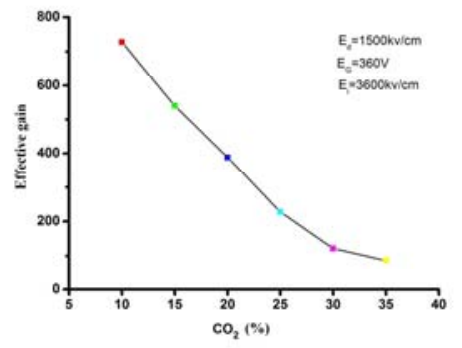

Fig.9. Gas mixture proportion of the electronic effective gain

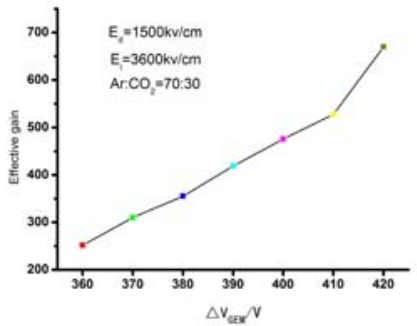

Fig.10. Single GEM effective gain with $\triangle$ VGEM various curv

However, if we respectively set the electric field of fixed drift area and GEM membrane to $3.6 \mathrm{kV} / \mathrm{cm}$ and $360 \mathrm{~V}$, while keep the ratio for $\mathrm{Ar} / \mathrm{CO}_{2}$ unchanged(70/30), we can observe that the effective gain will increase at first and then decrease, as sketched in Fig.11. From this diagram, we can learn that the maximum voltage is about $2-3 \mathrm{kV} / \mathrm{cm}$. We can explain this on the basis of principle of GEM: After X rays entering into the detector, It will produce thousands of electrons during the process of colliding with Ar atom, and eventually those electrons will pass through the GEM micro-porous membrane along potential line in drift field.

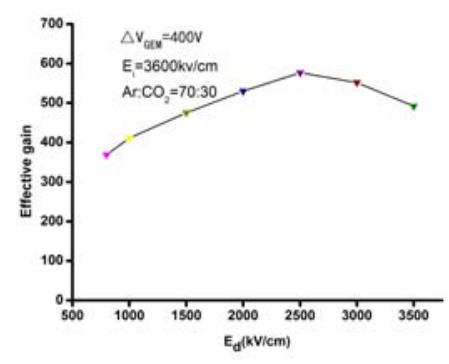

Fig.11. Effective gain of single GEM with Ed with the Ei various curve

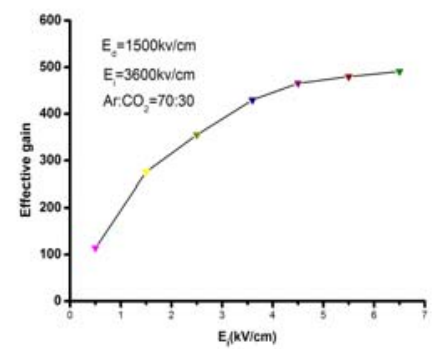

Fig.12. Effective gain of single GEM various curve

While, if the electric field in drift field becomes greater, electrons will gain more energy, and secondary electrons will also be generated and collected due to multiplication avalanche. However, when the electric field intensity continues to increasing, the electric field line will be terminated on the upper of GEM film. This phenomenon may eventually lead to scattering the GEM microspores' electric field. So from this perspective, there will be a number of primary ionization electrons collected by copper electrode in GEM film, which will consequently give rise to a significant reduction in secondary electrons and bad magnification effect ${ }^{[15]}$.

Last but not least, there is also another situation: If we kept the ratio for $\mathrm{Ar} / \mathrm{CO}_{2}$ (70/30) and electric field in GEM membrane $(360 \mathrm{~V})$ unchanged while set the electric field of fixed drift region at $1.0 \mathrm{kV} / \mathrm{cm}$, the effective gain will increases with the increasing voltage(see Fig.12). In this situation, we should take into account the primary and secondary electrons. When both of them go through microspores, they will be collected by electrodes. With the increase of Voltage, the electronic forces will be enhanced and absorbed electrons in drift field will getting less. Thereby, more collected electrons and higher effective gain will be emerged. However, if the electric field is still added to some extent, the electrons loss can be neglected. On this occasion, we can deem all of ionized electrons are collected, and the effective gain tends to a saturation degree.

\section{CONCLUSION}

In conclusion, this paper underlines the calculate process of internal detector electric field intensity and potential of the geometric model by using the Maxwell V11. And we also successfully simulated the process of electronic avalanche via Garfield program. During the simulation process, many factors that may lead to changing gas effectively gain such as the ratio for mixed gas, the variable drift and collected field, and the unstable voltage in GEM membrane are also taken into serious consideration. Those achievements can provide an important reference data for further work such as the GEM detector for effective gain, energy resolution, and spatial experiment.

\section{ACKNOWLEDGMENT}

*Project supported by the Natural Science Foundation of China (Grant No. 11104156), and the plan of scientific and technological projects of Jiangxi Province (Grant No. 
KJLD12076), the Open Foundation of Engineering Research Center of Nuclear Technology Application, Ministry of Education (Grant No.HJSJYB2011-05)

\section{CORRESPONDING AUTHOR}

Corresponding author.

Tel.: +86818970825192 .

E-mail address: xingxing_fz@sina.com, liuyb01@mails.tsinghua.edu.cn.

\section{REFERENCES}

[1] Xie Yigang. Review of gas detector. Modern Physics. 2011. Vol23(4):23-28)

[2] Wang FY,Sun ZJ,Huang GM, et al. M.C. Simulation of GEM Neutron Beam Monitor. The Fifteenth National Conference on Nuclear Electronics and Nuclear Detection Technology Symposium,2010:262-267

[3] LI Yu-Lan•PPT in CCAST•2007

[4] B. Yu et al•IEEE Nucl.Sci.Symp. 2005(Puerto Rico)

[5] LI Yu-Lan et al•Chinese Physics C $2008 \bullet$ Vol.32 $\bullet$ No.1

[6] G Bencivenni•Nucl.Instr.and Meth.A•2003•513•264

[7] A.Oed $\bullet$ Nuel.Instr.and Meth.A•525(2004)62-68

[8] B.Esposito et al•Nuel.Instr.and Meth.A•617(2010)155-157

[9] R.Cheehiketal•Nuel.Instr.andMeth.A•419(1998)423

[10] Zhao Bo, Zhang Hongliang, Ansoft12 application in engineering field

[11] Maxwell12[1].1 - Application of training. Institute of electrical engineering, Xi'an Jiaotong University

[12] http://www.maxwell.com/

[13] http://garfield.web.cern.ch/garfied

[14] Zhang Minglong, the development and performance testing of gas electron multiplier, Nuclear Electronics \& Detection Technology,2005,Vol(25)

[15] Su Qingfeng, Study on GEM detector counting performance, Shanghai Nonferrous Metals, 2005 